\title{
Generalized hydrodynamic equations for nematic liquid crystals
}

\author{
Tiezheng Qian ${ }^{1,2}$ and Ping Sheng ${ }^{1}$ \\ ${ }^{1}$ Department of Physics, The Hong Kong University of Science and Technology, Clear Water Bay, Kowloon, Hong Kong, China \\ ${ }^{2}$ Department of Physics, University of California at Berkeley, Berkeley, California 94720
}

(Received 9 July 1998)

\begin{abstract}
We present a formulation of the nematic liquid crystal hydrodynamics based on the tensor order parameter, with the spatial variations of both the director and the scalar (uniaxial and biaxial) order parameters explicitly taken into account. New length and time scales are shown to arise that can be important for the switching dynamics of thin cells subject to large external fields. The Ericksen-Leslie theory is shown to be a special limit of our formulation. [S1063-651X(98)13712-1]

PACS number(s): 61.30.- v, 83.70.Jr
\end{abstract}

\section{INTRODUCTION}

Nematic liquid crystals (NLC's) are at the same time similar and different from ordinary fluids. They are similar because both have no translational long-range order for the molecules. In addition, nematic liquid crystals possess no static shear modulus and can therefore flow like a fluid. However, NLC's have long-range orientational order, which gives rise to many of the special optical properties which are the basis of liquid crystal displays. The existence of the orientational order means that locally, the alignment of the NLC's is characterized by a director $\mathbf{n}$, the local axis of uniaxial orientational order. Based on symmetry and elastic energy considerations, a continuum elastic theory of NLC's has been developed by Oseen [1], Zocher [2], and Frank [3]. The incorporation of the orientational degree of freedom in the hydrodynamics of NLC's was subsequently developed by Ericksen [4] and Leslie [5]. They formulated the general conservation laws of fluid mechanics for NLC's and derived the hydrodynamic equations governing the time evolution of the director field $\mathbf{n}(\mathbf{r})$ and the velocity field $\mathbf{v}(\mathbf{r})$. The EricksenLeslie (EL) theory has been widely and successfully used in explaining various dynamic properties of thermotropic NLC's.

In addition to the director $\mathbf{n}$, which gives the local axis of uniaxial symmetry, the NLC's are also characterized by the degree of local orientational order $S(\mathbf{r})$. By noticing that NLC's do not distinguish the head and tail of the director $\mathbf{n}$, and by taking into account the degree of orientational order $S(\mathbf{r})$, de Gennes [6] has shown that the order parameter of NLC's is a second rank traceless symmetric tensor $\overleftrightarrow{Q}$. While the continuum elastic theory based on the director description of NLC is sufficient for many field-induced liquid crystal behaviors, it has been shown that the consideration of substrate-nematic interaction and/or interaction with strong electric or magnetic field would require the more complete theoretical framework based on the tensor order parameter. The Landau-de Gennes ( $\mathrm{LdG}$ ) [7] theory has been instrumental in explaining and predicting various static phenomena, such as surface wetting [8], surface-induced bulk alignment $[9,10]$, and defect core structure [11-13], all involving the fast spatial variations of liquid crystalline order (LCO), a term used here to denote both $\mathbf{n}$ and $S$ as expressed through the tensor order parameter $\stackrel{\leftrightarrow}{Q}$. Recently, it has been observed that the polyimide-coated substrates can induce a boundary layer of strong biaxial character, describable only through the tensor order parameter [9]. In addition, the prediction of the bulk orientational order through the LdG theory shows good agreement with the experiment. The use of a tensor order parameter is therefore not only necessary, but also sufficiently accurate.

The purpose of this work is to explore the role of $\mathrm{LCO}$, as expressed by the tensor order parameter $\overleftrightarrow{Q}$, in hydrodynamic processes. We adopt the general framework developed by Ericksen and Leslie, meanwhile applying the LdG free energy to present a hydrodynamics formulation that can be used to study the dynamic behavior. We identify new length and time scales associated with the spatially and temporally varying LCO in generic hydrodynamic processes. Illustrative numerical results are given. It is shown that the EL theory corresponds to the long-range, slow-motion limit of the general formulation. We note that the EL theory has been rewritten in the tensorial context but with $S(\mathbf{r})$ treated as a constant $[14,15]$. Our formulation should be distinguished from such treatment since we take into account the variations of both $S(\mathbf{r})$ and $\mathbf{n}(r)$, as well as the coupling between the two and/or the biaxial order.

\section{STATEMENT OF RESULTS}

The LdG free energy density is given by [7]

$$
\begin{aligned}
\mathcal{F}_{L d G}= & \frac{1}{2}\left(\alpha Q_{i j}^{2}+L_{1} Q_{i j, k}^{2}+L_{2} Q_{i j, j} Q_{i k, k}\right) \\
& +\frac{4 \pi}{P} L_{1} \epsilon_{i j k} Q_{i l} \partial_{j} Q_{k l}-\beta Q_{i j} Q_{j k} Q_{k i}+\gamma\left(Q_{i j}^{2}\right)^{2} .
\end{aligned}
$$

Here $\alpha, L_{1}, L_{2}, \beta, \gamma$, and $P$ are phenomenological material constants, $\epsilon_{i j k}$ is the Levi-Civita symbol, the indices $i, j, k$ run from 1 to 3 , summation over repeated indices is implied, and the comma in the subscript means derivative with respect to the spatial coordinate that follows. We consider incompressible NLC's obeying $\partial_{k} v_{k}=0$. The central results of this work are as follows. The hydrodynamic equations for the flow and LCO are 


$$
\begin{array}{r}
\rho \frac{d v_{i}}{d t}=\partial_{j}\left(-p \delta_{j i}+\sigma_{j i}^{d}+\sigma_{j i}^{f}+\sigma_{j i}^{\prime}\right), \\
J \ddot{Q}_{i j}=h_{i j}+h_{i j}^{\prime}-\lambda \delta_{i j}-\epsilon_{i j k} \lambda_{k},
\end{array}
$$

Here $\rho$ is the NLC density, $p$ denotes the pressure, $J$ is the moment of inertial density, usually negligible, $\lambda$ and $\lambda_{k}$ are the Lagrange multipliers determined by requiring $\operatorname{Tr} \overleftrightarrow{Q}=0$ and $Q_{i j}=Q_{j i}$, while $d / d t$ is the total time derivative $\partial / \partial t$ $+\mathbf{v} \cdot \nabla$. The distortion stress $\boldsymbol{\sigma}^{d}$ and elastic molecular field $\mathbf{h}$ are determined by the LdG free energy:

$$
\begin{gathered}
\sigma_{j i}^{d}=-\frac{\partial \mathcal{F}_{L d G}}{\partial\left(\partial_{j} Q_{\alpha \beta}\right)} \partial_{i} Q_{\alpha \beta}, \\
h_{i j}=-\frac{\partial \mathcal{F}_{L d G}}{\partial Q_{i j}}+\partial_{k} \frac{\partial \mathcal{F}_{L d G}}{\partial\left(\partial_{k} Q_{i j}\right)} .
\end{gathered}
$$

In the presence of electric field $\mathbf{E}$, there is a field-induced free energy density $\mathcal{F}_{f}=-(1 / 8 \pi) \mathbf{D} \cdot \mathbf{E}$, where $\mathbf{D}=\stackrel{\leftrightarrow}{\epsilon} \mathbf{E}$ is the electric displacement field, and $\stackrel{\leftrightarrow}{\epsilon}=\frac{2}{3} \epsilon_{a}^{m} \overleftrightarrow{Q}+\bar{\epsilon} I$ is the dielectric tensor with $\epsilon_{a}^{m}$ being the maximum dielectric anisotropy and $\bar{\epsilon}=\operatorname{Tr} \stackrel{\leftrightarrow}{\epsilon} / 3$. Here the sign in $-(1 / 8 \pi) \mathbf{D} \cdot \mathbf{E}$ means that $\mathcal{F}_{f}$ takes the electric field (or potential) as thermodynamical coordinate. The field-induced stress $\boldsymbol{\sigma}^{f}$ is given by

$$
\sigma_{j i}^{f}=\frac{1}{4 \pi} D_{j} E_{i}-\frac{1}{8 \pi}\left(D_{k} E_{k}-\rho E_{\mu} E_{\nu} \frac{\partial \epsilon_{\mu \nu}}{\partial \rho}\right) \delta_{j i},
$$

and $\mathbf{h}$ is reexpressed by adding the field-induced molecular field $(1 / 12 \pi) \epsilon_{a}^{m} E_{i} E_{j}$ to the right-hand side (RHS) of Eq. (5). The viscous stress $\boldsymbol{\sigma}^{\prime}$ and viscous molecular field $\mathbf{h}^{\prime}$ are given by

$$
\begin{gathered}
\sigma_{\alpha \beta}^{\prime}=\beta_{1} Q_{\alpha \beta} Q_{\mu \nu} A_{\mu \nu}+\beta_{4} A_{\alpha \beta}+\beta_{5} Q_{\alpha \mu} A_{\mu \beta}+\beta_{6} Q_{\beta \mu} A_{\mu \alpha} \\
+\frac{1}{2} \mu_{2} \mathcal{N}_{\alpha \beta}-\mu_{1} Q_{\alpha \mu} \mathcal{N}_{\mu \beta}+\mu_{1} Q_{\beta \mu} \mathcal{N}_{\mu \alpha} \\
-h_{\alpha \beta}^{\prime}=\frac{1}{2} \mu_{2} A_{\alpha \beta}+\mu_{1} \mathcal{N}_{\alpha \beta}
\end{gathered}
$$

where $\beta_{1}, \beta_{4}, \beta_{5}, \beta_{6}, \mu_{1}$, and $\mu_{2}$ are viscosity coefficients with $\beta_{6}-\beta_{5}=\mu_{2}, A_{j i}=\frac{1}{2}\left(\partial_{j} v_{i}+\partial_{i} v_{j}\right)$ is the symmetric part of the velocity gradient tensor, while $\mathcal{N}_{j i}=\partial Q_{j i} / \partial t$ $+v_{k} \partial_{k} Q_{j i}-\epsilon_{j \alpha \beta} \omega_{\alpha} Q_{\beta i}-\epsilon_{i \alpha \beta} \omega_{\alpha} Q_{j \beta}$ is the time rate of change of $Q_{j i}$ with respect to the background fluid angular velocity $\boldsymbol{\omega}=\frac{1}{2} \nabla \times \mathbf{v}$.

There are intrinsic length and time scales that emerge from the LdG free energy. We choose the length scale $\Delta$ $=\sqrt{L C / B^{2}}$, where $B=3 \beta / 4, \quad C=9 \gamma / 4$, and $L=3 L_{1} / 4$ $+L_{2} / 2$, as the characterisitic length unit to describe the spatial variations of $Q_{j i}$. Correspondingly, the time scale is $\tau$ $=\eta \Delta^{2} / L$ where $\eta$ is some representative viscosity coefficient. For most NLC's, $\Delta \sim 50 \AA$ and $\tau \sim 0.1 \mu \mathrm{s}$. In addition to these intrinsic scales, there are also the length and time scales that arise from external imposed parameters, such as those in the EL theory. Consider a liquid crystal cell of thickness $d$ with parallel, uniform substrates imposing fixed surface alignment conditions. The EL theory gives $d$ as the length scale and $\tau_{0} \sim \eta d^{2} / K$ as the time scale, where $K$ $\sim L S^{2}$ is a Frank elastic constant in the continuum theory. In the presence of applied voltage $V$, the field-induced correlation length is given by $\xi_{E} \sim \sqrt{4 \pi K / \epsilon_{a} E^{2}}$ ( $\epsilon_{a}$ is the dielectric anisotropy and $E=V / d$ is the field strength) and the associated time scale becomes $\tau_{E} \sim \eta \xi_{E}^{2} / K$. In our formulation, the EL theory is recovered when $\Delta \ll \xi_{E} \leqslant d$ and $\tau \ll \tau_{E} \leqslant \tau_{0}$, i.e., in the limit of slow spatial and temporal variations. In fact, the EL theory can be obtained from our formulation when $Q_{i j}$ is written as

$$
Q_{i j}(\mathbf{r})=S_{0}\left[3 n_{i}(\mathbf{r}) n_{j}(\mathbf{r})-\delta_{i j}\right] / 2,
$$

i.e., the orientational order is treated as a frozen constant. However, that means the dynamic behavior of $S(\mathbf{r})$, and those involving the coupled evolution of $S(\mathbf{r})$ and $\mathbf{n}(r)$, cannot be described within the EL theory.

We have carried out numerical calculation for a cell of $d \sim 1 \mu \mathrm{m}$ filled by a NLC of large $\epsilon_{a} \sim 15$, with homogeneous substrate alignment conditions. We first minimize the LdG free energy to obtain the equilibrium NLC configuration under a large holding voltage $(\sim 20 \mathrm{~V})$. Biaxial ordering in the boundary layer is noted. The hydrodynamic equations (2) and (3) are then solved to show the backflow effect $[16,17]$ after the voltage is switched off. It is seen that the large elastic energy stored in the biaxial boundary layers results in a very fast orientational relaxation close to the NLCsubstrate interfaces. Because of the coupling between the translational and orientational motions, a large flow field is induced. The length and time scales $\Delta$ and $\tau$ are clearly manifest in the resulting dynamic behavior.

The paper is organized as follows. In Sec. III, the derivation of the hydrodynamics equations, based on the tensor order parameter, is presented. In Sec. IV, the new length and time scales of our formulation are discussed, and the EL theory is obtained as a limit of our equations. In Sec. V, the numerical results are given to illustrate the hydrodynamic effects of LCO in thin cells subject to strong fields. We conclude in Sec. VI with a discussion of implications for future work.

\section{EQUATIONS OF HYDRODYNAMICS}

In this section we derive the hydrodynamic equations for NLC's by using the LdG free energy to define the nondissipative molecular field and stress. The viscous molecular field and stress are then introduced through the consideration of entropy production in a dissipative flowing nematic. The two independent fluxes for friction are identified by using the conservation of angular momentum, and explicit expressions for the viscous forces are obtained, leading directly to Eqs. (2) and (3). Field-induced effects are included in the derivation.

\section{A. Free energy and hydrostatics}

The LdG free energy $F_{L d G}=\int \mathcal{F}_{L d G} d \mathbf{r}$ is a functional of $Q_{i j}(\mathbf{r})$ and $Q_{i j, k}(\mathbf{r})$. The NLC can be "virtually" distorted by either a rotation of molecular alignment: $Q_{i j}(\mathbf{r})$ $\rightarrow Q_{i j}^{\prime}(\mathbf{r})$, or a displacement while keeping its orientation fixed: $\mathbf{r} \rightarrow \mathbf{r}^{\prime}=\mathbf{r}+\mathbf{u}(\mathbf{r}), Q_{i j}(\mathbf{r}) \rightarrow Q_{i j}^{\prime}\left(\mathbf{r}^{\prime}\right)=Q_{i j}(\mathbf{r})$. The elastic molecular field $\mathbf{h}$, as defined by Eq. (5), results directly from the virtual orientational distortion. The equilibrium state is given by the order parameter configuration that mini- 
mizes the free energy functional $\int d \mathbf{r} \mathcal{F}_{L d G}$. As $\overleftrightarrow{Q}$ only has five independent components, the optimal configuration satisfies $h_{i j}=\lambda \delta_{i j}+\epsilon_{i j k} \lambda_{k}$, where the four $\lambda$ and $\lambda_{k}$ are Lagrange multipliers due to the constraints $\operatorname{Tr} \overleftrightarrow{Q}=0$ and $Q_{i j}=Q_{j i}$. On the other hand, the distortion stress $\boldsymbol{\sigma}^{d}$ is defined by $\delta F_{L d G}=\int \sigma_{j i}^{d} \partial_{j} u_{i} d \mathbf{r}$. Here the change in free energy is caused by a change of $Q_{i j, k}: \partial_{k}^{\prime} Q_{i j}^{\prime}-\partial_{k} Q_{i j} \simeq$ $-\partial_{\alpha} Q_{i j} \partial_{k} u_{\alpha}$, and $\delta F_{L d G}$ is given by

$$
\delta F_{L d G}=\int d \mathbf{r} \frac{\partial \mathcal{F}_{L d G}}{\partial Q_{i j, k}}\left(-\partial_{\alpha} Q_{i j} \partial_{k} u_{\alpha}\right),
$$

from which we obtain Eq. (4). In the presence of an electric field $\mathbf{E}$, the total free energy density becomes $f=\mathcal{F}_{L d G}$ $+\mathcal{F}_{f}$, where the field-induced energy density $\mathcal{F}_{f}$ is given by $-(1 / 8 \pi) \epsilon_{i j} E_{i} E_{j}$. Accordingly, the molecular field becomes

$$
h_{j i}=\Lambda_{j i}+\partial_{\mu} \Pi_{\mu j i}+\frac{\epsilon_{a}^{m}}{12 \pi} E_{j} E_{i},
$$

where $\Lambda_{j i}=-\partial \mathcal{F}_{L d G} / \partial Q_{j i}$ and $\Pi_{\mu j i}=\partial \mathcal{F}_{L d G} / \partial Q_{j i, \mu}$. The field-induced stress $\boldsymbol{\sigma}^{f}$, defined by $\delta \mathcal{F}_{f}=\sigma_{j i}^{f} \partial_{j} u_{i}$, is consequently given by Eq. (6) [18].

The LdG free energy is invariant under a rigid rotation of the nematic. That is, $\delta F_{L d G}=0$ if $u_{i}(\mathbf{r})=\epsilon_{i j k} \delta \Theta_{j} r_{k}, \delta Q_{i j}$ $=\epsilon_{i \alpha \beta} \delta \Theta_{\alpha} Q_{\beta j}+\epsilon_{j \alpha \beta} \delta \Theta_{\alpha} Q_{i \beta}$, where $\delta \Theta$ is a small rotation angle. $\delta F_{L d G}$ can be expressed as

$$
\delta \Theta_{\mu} \mathcal{L}_{\mu}=0 .
$$

As $\delta \Theta_{\mu}$ is arbitrary, we have

$$
\begin{aligned}
\mathcal{L}_{\mu}= & \int d \mathbf{r}\left\{\epsilon_{j i \mu} \sigma_{j i}^{d}-\Lambda_{j i}\left(\epsilon_{j \mu k} Q_{k i}+\epsilon_{i \mu k} Q_{j k}\right)\right. \\
& \left.+\Pi_{\nu j i}\left(\epsilon_{j \mu k} Q_{k i, \nu}+\epsilon_{i \mu k} Q_{j k, \nu}\right)\right\}=0,
\end{aligned}
$$

which essentially expresses angular momentum conservation of the system, and shows that $\boldsymbol{\sigma}^{d}$ and $\mathbf{h}$ are not independent. Equation (11) can also take the form

$$
\begin{aligned}
& \int d \mathbf{r}\left\{\epsilon_{i j k} \sigma_{j k}^{d}+\partial_{\nu}\left[\epsilon_{i j k}\left(Q_{j \mu} \Pi_{\nu k \mu}+Q_{\mu j} \Pi_{\nu \mu k}\right)\right]\right\} \\
& =\int d \mathbf{r}\left\{\epsilon_{i j k}\left[Q_{j \mu}\left(\Lambda_{k \mu}+\partial_{\nu} \Pi_{\nu k \mu}\right)+Q_{\mu j}\left(\Lambda_{\mu k}+\partial_{\nu} \Pi_{\nu \mu k}\right)\right]\right\} \\
& =\int d \mathbf{r}\left\{\epsilon_{i j k}\left(Q_{j \mu} h_{k \mu}+Q_{\mu j} h_{\mu k}\right)-\frac{\epsilon_{a}^{m}}{12 \pi} \epsilon_{i j k}\left(Q_{j \mu} E_{k} E_{\mu}\right.\right. \\
& \left.\left.\quad+Q_{\mu j} E_{\mu} E_{k}\right)\right\}
\end{aligned}
$$

in which we replace $\Lambda_{\alpha \beta}+\partial_{\nu} \Pi_{\nu \alpha \beta}$ by $h_{\alpha \beta}-\epsilon_{a}^{m} E_{\alpha} E_{\beta} / 12 \pi$ using Eq. (10). This leads to the term $\int d \mathbf{r}\left\{-\left(\epsilon_{a}^{m} / 12 \pi\right) \epsilon_{i j k}\left(Q_{j \mu} E_{k} E_{\mu}+Q_{\mu j} E_{\mu} E_{k}\right)\right\}$ explicitly depending on $\mathbf{E}$ in the RHS of the above equation. Then using $\overleftrightarrow{\epsilon}=\frac{2}{3} \epsilon_{a}^{m} \overleftrightarrow{Q}+\bar{\epsilon} I$ for the dielectric tensor, $-\left(\epsilon_{a}^{m} / 12 \pi\right) \epsilon_{i j k}\left(Q_{j \mu} E_{k} E_{\mu}+Q_{\mu j} E_{\mu} E_{k}\right)$ is found to be equal to $-(1 / 4 \pi) \epsilon_{i j k} D_{j} E_{k}$, which is simply related to $\boldsymbol{\sigma}^{f}$ by $-(1 / 4 \pi) \epsilon_{i j k} D_{j} E_{k}=-\epsilon_{i j k} \sigma_{j k}^{f}$. Thus, Eq. (11) can be expressed as

$$
\begin{aligned}
& \int d \mathbf{r}\left\{\epsilon_{i j k}\left(\sigma_{j k}^{d}+\sigma_{j k}^{f}\right)+\partial_{\nu}\left[\epsilon_{i j k}\left(Q_{j \mu} \Pi_{\nu k \mu}+Q_{\mu j} \Pi_{\nu \mu k}\right)\right]\right\} \\
& =\int d \mathbf{r}\left\{\epsilon_{i j k}\left(Q_{j \mu} h_{k \mu}+Q_{\mu j} h_{\mu k}\right)\right\},
\end{aligned}
$$

from which we obtain

$$
\begin{aligned}
& \int \epsilon_{i j k} r_{j} d S_{\mu}\left(\sigma_{\mu k}^{d}+\sigma_{\mu k}^{f}\right)+\int \epsilon_{i j k}\left(Q_{j \mu} d S_{\nu} \Pi_{\nu k \mu}\right. \\
& \left.\quad+Q_{\mu j} d S_{\nu} \Pi_{\nu \mu k}\right) \\
& =\int \epsilon_{i j k}\left\{r_{j} \partial_{\mu}\left(\sigma_{\mu k}^{d}+\sigma_{\mu k}^{f}\right)+\left(Q_{j \mu} h_{k \mu}+Q_{\mu j} h_{\mu k}\right)\right\} d \mathbf{r},
\end{aligned}
$$

by using $\epsilon_{i j k}\left(\sigma_{j k}^{d}+\sigma_{j k}^{f}\right)=\epsilon_{i j k} \partial_{\mu}\left[r_{j}\left(\sigma_{\mu k}^{d}+\sigma_{\mu k}^{f}\right)\right]$ $-\epsilon_{i j k} r_{j} \partial_{\mu}\left(\sigma_{\mu k}^{d}+\sigma_{\mu k}^{f}\right)$. Here $d \mathbf{S}$ is the vectorial surface element on the boundary. It is clearly seen that the body torques are externally supplied by the surface torques. In Eq. (12), since the appearance of $\left(\sigma_{j i}^{d}+\sigma_{j i}^{f}\right)$ is always in conjuction with $\epsilon_{i j k}$, which is totally antisymmetric, it follows that the result will not be altered by replacing $\sigma_{j i}^{d}+\sigma_{j i}^{f}$ by $\sigma_{j i}^{d}+\sigma_{j i}^{f}$ $-p \delta_{j i}$, since $-p \delta_{j i}$ is symmetric. However, in equilibrium $\partial_{j}\left(-p \delta_{j i}+\sigma_{j i}^{d}+\sigma_{j i}^{f}\right)=0$ and $h_{i j}=\lambda \delta_{i j}+\epsilon_{i j k} \lambda_{k}$, therefore the integrant in the RHS of Eq. (12) vanishes. That is, the body torque equal to zero, consistent with the requirement of angular momentum conservation.

\section{B. Dissipative dynamics and entropy source}

With $\boldsymbol{\sigma}^{d}, \boldsymbol{\sigma}^{f}$, and $\mathbf{h}$ defined from the free energy expression, we are ready to write down the dynamic equations by introducing the viscous stress $\boldsymbol{\sigma}^{\prime}$ and the viscous molecular field $\mathbf{h}^{\prime}$ which are responsible for entropy production in flowing nematics. Knowledge of the viscous stress means the dynamical equation, expressed as conservation of linear momentum, may be written as

$$
\frac{d}{d t} \int \rho v_{i} d \mathbf{r}=\int d S_{j} \sigma_{j i},
$$

where the total stress $\sigma_{j i}=-p \delta_{j i}+\sigma_{j i}^{d}+\sigma_{j i}^{f}+\sigma_{j i}^{\prime}$. The last term, $\sigma_{j i}^{\prime}$, represents the viscous stress tensor, which is to be derived. The time evolution of LCO is governed by the angular momentum equation

$$
\begin{aligned}
& \frac{d}{d t} \int J \epsilon_{i j k}\left(Q_{j \mu} \dot{Q}_{k \mu}+Q_{\mu j} \dot{Q}_{\mu k}\right) d \mathbf{r} \\
& \quad=\int\left[Q_{j \mu}\left(h_{k \mu}+h_{k \mu}^{\prime}\right)+Q_{\mu j}\left(h_{\mu k}+h_{\mu k}^{\prime}\right)\right] d \mathbf{r},
\end{aligned}
$$

where the viscous molecular field $\mathbf{h}^{\prime}$ is introduced as a friction force with respect to the rotational degree of freedom, also to be derived. The differential equations corresponding to Eqs. (13) and (14) are Eqs. (2) and (3) stated in Sec. II. Equation (3) is actually the tensorial analogue of the vectorial Oseen equation [see Eq. (A4) in Appendix A] of the EL theory. That relation will be dicussed in Sec. IV and Appendix B. 
The entropy production in an isothermal flowing nematic is defined by the thermodynamic relation

$$
\dot{F}=\dot{\mathcal{W}}+\dot{\mathcal{Q}}-T \dot{\mathcal{S}}=\dot{\mathcal{W}}-T \dot{\Sigma},
$$

where $\mathcal{S}$ denotes entropy, and $T \dot{\Sigma}$ denotes the dissipative part of entropy production. Written out explicitly, we have

$$
\begin{aligned}
T \dot{\Sigma}= & -\frac{d}{d t} \int\left(\frac{1}{2} \rho \mathbf{v}^{2}+\frac{1}{2} J \dot{Q}_{\alpha \beta}^{2}+\mathcal{F}_{L d G}+\mathcal{F}_{f}\right) \\
& +\int d S_{\mu}\left(\Pi_{\mu \alpha \beta} \dot{Q}_{\alpha \beta}+\sigma_{\mu \nu} v_{\nu}\right),
\end{aligned}
$$

where the first integral is the decreasing rate of the total free energy $(-\dot{F})$ and the second integral is the rate of external work $(\dot{\mathcal{W}})$ done by the surface forces. Substituting the dynamic equations (2) and (3) into Eq. (15) yields

$$
T \dot{\Sigma}=\int d \mathbf{r}\left\{\sigma_{j i}^{\prime} A_{j i}-h_{j i}^{\prime} \dot{Q}_{j i}\right\}
$$

where $A_{j i}$ has been defined previously in relation to Eqs. (7) and (8). In Eq. (16), while we have explicitly identified $\boldsymbol{\sigma}^{\prime}$ and $\mathbf{h}^{\prime}$, yet the two quantities are correlated through angular momentum conservation. To make this clear, we note that the rate of change of angular momentum may be expressed as

$$
\begin{gathered}
\int \epsilon_{i j k} r_{j} d S_{\mu} \sigma_{\mu k}+\int \epsilon_{i j k}\left(Q_{\alpha j} d S_{\mu} \Pi_{\mu \alpha k}+Q_{j \alpha} d S_{\mu} \Pi_{\mu k \alpha}\right) \\
=\frac{d}{d t} \int\left\{\epsilon_{i j k} \rho r_{j} v_{k}+\epsilon_{i j k} J\left(Q_{\mu j} \dot{Q}_{\mu k}+Q_{j \mu} \dot{Q}_{k \mu}\right)\right\} d \mathbf{r} .
\end{gathered}
$$

Using Eqs. (2) and (3), we may express the RHS of Eq. (17) as

$$
\int \epsilon_{i j k}\left\{r_{j} \partial_{\mu} \sigma_{\mu k}+\left[Q_{j \mu}\left(h_{k \mu}+h_{k \mu}^{\prime}\right)+Q_{\mu j}\left(h_{\mu k}+h_{\mu k}^{\prime}\right)\right]\right\} d \mathbf{r} .
$$

We thus see that the sum of body torques, given by $\partial_{\mu} \sigma_{\mu i}$ and $\mathbf{h}+\mathbf{h}^{\prime}$, equals the sum of surface torques. However, the sum of body torques exerted by $\partial_{\mu}\left(\sigma_{\mu i}^{d}+\sigma_{\mu i}^{f}\right)$ and $\mathbf{h}$ must equal to the sum of nondissipative surface torques, as stated by Eq. (12). Subtracting Eq. (12) from (17), we obtain

$$
\epsilon_{i j k} \partial_{\mu}\left(r_{j} \sigma_{\mu k}^{\prime}\right)=\epsilon_{i j k} r_{j} \partial_{u} \sigma_{\mu k}^{\prime}+\epsilon_{i j k}\left(Q_{\mu j} h_{\mu k}^{\prime}+Q_{j \mu} h_{k \mu}^{\prime}\right),
$$

which simply means that the viscous body torques from $\partial_{\mu} \sigma_{\mu i}^{\prime}$ and $\mathbf{h}^{\prime}$ are supplied by the surface torque from $d S_{\mu} \sigma_{\mu i}^{\prime}$. Substituting Eq. (18) into (16), we obtain the entropy production expression

$$
T \dot{\Sigma}=\int d \mathbf{r}\left\{\sigma_{j i}^{\prime s} A_{j i}-h_{j i}^{\prime} \mathcal{N}_{j i}\right\}
$$

where $\sigma_{j i}^{\prime s}$ is the symmetric part of $\sigma_{j i}^{\prime}$. The two sets of independent fluxes $A_{i j}$ and $\mathcal{N}_{i j}$ are thus identified as defined previously in relation to Eqs. (7) and (8), and the laws of friction can be explicitly expressed as

$$
\begin{gathered}
\sigma_{\alpha \beta}^{\prime s}=\beta_{1} Q_{\alpha \beta} Q_{\mu \nu} A_{\mu \nu}+\beta_{4} A_{\alpha \beta}+\frac{1}{2}\left(\beta_{5}+\beta_{6}\right)\left(Q_{\alpha \mu} A_{\mu \beta}\right. \\
\left.+Q_{\beta \mu} A_{\mu \alpha}\right)+\frac{1}{2} \mu_{2} \mathcal{N}_{\alpha \beta} \\
\quad-h_{\alpha \beta}^{\prime}=\frac{1}{2} \mu_{2}^{\prime} A_{\alpha \beta}+\mu_{1} \mathcal{N}_{\alpha \beta}
\end{gathered}
$$

where $\beta_{1}, \beta_{4}, \frac{1}{2}\left(\beta_{5}+\beta_{6}\right), \mu_{2}, \mu_{2}^{\prime}$, and $\mu_{1}$ are independent viscosity coefficients. According to the Onsager theorem [19], $\mu_{2}=\mu_{2}^{\prime}$. Here the expressions for $\sigma_{\alpha \beta}^{\prime s}$ and $-h_{\alpha \beta}^{\prime}$ keep the lowest-order terms in $Q_{i j}$ so as to avoid the definition of a large number of viscosity coefficients. They can be related to the corresponding quantities in the EL formulation. This is elaborated in Sec. IV and Appendix B. Using $\sigma_{\alpha \beta}^{\prime}=\sigma_{\alpha \beta}^{\prime s}$ $+\frac{1}{2} \epsilon_{\mu \alpha \beta} \epsilon_{\mu i j} \sigma_{i j}^{\prime}$ and Eq. (18), we obtain $\sigma_{\alpha \beta}^{\prime}$ as given by Eq. (7). With the explicit expressions obtained for all the stresses and molecular fields, the derivation of the nematodynamics equations is thus complete.

\section{DISCUSSION}

\section{A. Intrinsic length and time scales}

The short-range, fast variations of LCO can be induced by surface interaction potentials, strong fields, and/or tight geometric confinements. The characteristic distances associated with such variations are determined by the NLCs' elastic correlation lengths, which can be defined from $\mathcal{F}_{L d G}$. In Sec. II we define $\Delta$ as the characteristic length unit to describe the spatial variations of $Q_{j i}$. Since the inertial effects are usually negligible, the dynamic equations (2) and (3) are essentially the balance equations between elastic forces and viscous forces, and the intrinsic time scale $\tau$ corresponding to the NLCs' length scale $\Delta$ is determined by such viscoelastic effects. Since $\mathbf{h}, \boldsymbol{\sigma}^{d} \sim L / \Delta^{2}$ and $\mathbf{h}^{\prime}, \boldsymbol{\sigma}^{\prime} \sim \eta / \tau$, setting $L / \Delta^{2}$ $=\eta / \tau$ leads to $\tau \sim \eta \Delta^{2} / L$, where $\eta$ denotes some typical viscosity coefficient. Consider the NLC confined in a cell of thickness $d$ with applied voltage $V$. The EL theory defines two other sets of length and time scales. (For a brief review of the EL theory, see Appendix A.) The Frank free energy density $\mathcal{F}_{F}$, given by Eq. (A1) in Appendix A, leads to a correlation length $\rightarrow \infty$ [20], and the cell thickness $d$ becomes the only length scale. According to the EL theory, the corresponding time scale $\tau_{0}$ is determined by the balance between $\mathbf{g}, \tilde{\boldsymbol{\sigma}}^{d} \sim K / d^{2}$ and $\mathbf{g}^{\prime}, \tilde{\boldsymbol{\sigma}}^{\prime} \sim \eta / \tau_{0}$. That means $\tau_{0} \sim \eta d^{2} / K$. In the presence of electric field applied perpendicular to the substrates, $\mathcal{F}_{F}$ and the field-induced free energy density $-(1 / 8 \pi) \epsilon_{a}(\mathbf{n} \cdot \mathbf{E})^{2}$ together lead to a finite correlation length $\xi_{E} \sim \sqrt{4 \pi K d^{2} / \epsilon_{a} V^{2}}$ where $V$ is the applied voltage. The corresponding time scale $\tau_{E}$, determined by the balance between $\mathbf{g}, \tilde{\boldsymbol{\sigma}}^{d} \sim K / \xi_{E}^{2}$ and $\mathbf{g}^{\prime}, \tilde{\boldsymbol{\sigma}}^{\prime} \sim \eta / \tau_{E}$, is thus $\sim \eta \xi_{E}^{2} / K$.

We emphasize that $d, \tau_{0}$ and $\xi_{E}, \tau_{E}$ are determined by the cell thickness and applied field while the new length and time scales $\Delta$ and $\tau$ are intrinsic parameters that can only be obtained by taking into account the elastic correlation of LCO. With $L \sim 10^{-6}$ dyn, $B \sim 0.5 \mathrm{~J} / \mathrm{cm}^{3}, C \sim 1.0 \mathrm{~J} / \mathrm{cm}^{3}$, and $\eta \sim 0.1 p$ (for 5CB), we have $\Delta \sim 50 \AA$ and $\tau$ $\sim 0.1 \mu \mathrm{s}$. For large $\epsilon_{a} \sim 15$ [4-n-pentyl-4' -cyanobiphenyl (5CB) has $\left.\epsilon_{a} \simeq 13\right], V \sim 20 \mathrm{~V}$ and $d \sim 1 \mu \mathrm{m}$, the fieldinduced correlation length $\xi_{E} \sim 100 \AA$, which is comparable to $\Delta$. Such high voltages can induce the spatial variations as well as the time evolutions of LCO with $\Delta$ and $\tau$ as the 
relevant length and time scales. However, for large thickness $d$, low voltage $V$, and small dielectric anisotropy $\epsilon_{a}, \Delta$ is much smaller than $d$ and $\xi_{E}$. In that limit, the $S(\mathbf{r})$ is effectively a frozen degree of freedom that cannot be activated. Consequently, the director field suffices for the description of time-evolving alignment state, and our formulation equations are reduced to the EL theory. This is shown below with more details.

\section{B. Adiabatic $S(r, t)$ and the Ericksen-Leslie theory}

It is straightforward to reduce our formulation to the director description by taking the limit expressed by Eq. (9). Here we focus on the role of $S$ in such limit. A detailed comparison can be found in Appendix B.

We first restrict our discussion to the uniaxial case. From $Q_{i j}=S\left(3 n_{i} n_{j}-\delta_{i j}\right) / 2, \mathcal{F}_{L d G}$ is reduced to

$$
\begin{aligned}
\mathcal{F}_{L d G}^{U}= & a\left(T-T^{*}\right) S^{2}-B S^{3}+C S^{4}+\frac{3}{4}\left(L_{1}+\frac{1}{6} L_{2}\right)[\nabla S]^{2} \\
& +\frac{3}{8} L_{2}[\mathbf{n} \cdot \nabla S]^{2}+\frac{3}{2} L_{2} S[\nabla \cdot \mathbf{n}][\mathbf{n} \cdot \nabla S] \\
& +\frac{3}{4} L_{2} S[\mathbf{n} \times \nabla \times \mathbf{n}] \cdot \nabla S+\frac{9}{4} S^{2}\left\{\left(L_{1}+\frac{1}{2} L_{2}\right)[\nabla \cdot \mathbf{n}]^{2}\right. \\
& \left.+L_{1}[\mathbf{n} \cdot \nabla \times \mathbf{n}]^{2}+\left(L_{1}+\frac{1}{2} L_{2}\right)[\mathbf{n} \times \nabla \times \mathbf{n}]^{2}\right\},
\end{aligned}
$$

where $a\left(T-T^{*}\right)=3 \alpha / 4, T^{*}$ is the supercooling temperature, $B=3 \beta / 4$, and $C=9 \gamma / 4$. Here the helical term is omitted for simplicity. In case $S=S_{0}$ with $S_{0}$ being the uniaxial order parameter that minimizes $a\left(T-T^{*}\right) S^{2}+B S^{3}+C S^{4}$, the free energy is effectively a functional of $\mathbf{n}(\mathbf{r})$ only, represented by the last three terms. The splay, twist, and bend distortions of $\mathbf{n}(\mathbf{r})$ are expressed as in the Frank theory. The Frank elastic constants, given by $K_{1}=\frac{9}{2} S^{2}\left(L_{1}+\frac{1}{2} L_{2}\right), K_{2}$ $=\frac{9}{2} S^{2} L_{1}$, and $K_{3}=\frac{9}{2} S^{2}\left(L_{1}+\frac{1}{2} L_{2}\right)$, are predicted to be proportional to $S^{2}$ [21], which has been experimentally verified by measuring the temperature dependence of $K_{i}$ and $S$.

The EL theory ignores both the spatial and temporal dependence of $S(\mathbf{r})$. This corresponds to Eq. (9) in which the uniaxial orientational order parameter $S$ is set equal to $S_{0}$. To justify such an approximation, it is necessary to examine the equation $Q_{i j}\left(h_{i j}+h_{i j}^{\prime}\right)=0$, obtained from Eq. (3) by neglecting the inertial term. This is actually the molecular field balance equation for the $S$ degree of freedom. To make this clear, we first note from the definition of $h_{i j}$ [Eq. (5)] that

$$
Q_{i j} h_{i j}=S\left\{-\frac{\partial \mathcal{F}_{L d G}^{U}}{\partial S}+\partial_{k} \frac{\partial \mathcal{F}_{L d G}^{U}}{\partial\left(\partial_{k} S\right)}\right\}-\frac{3 S}{2} \frac{\partial \mathcal{F}_{L d G}^{U}}{\partial\left(\partial_{k} Q_{i j}\right)} \partial_{k}\left(n_{i} n_{j}\right) .
$$

In the RHS of this equation, the second term is negligible compared with the first term if the characteristic distance $l_{\mathbf{n}}$ over which the director variations take place is much larger than $\Delta$, the characteristic distance for the spatial variations of $S$. An estimate of order of magnitude shows that in the RHS of Eq. (23), the first terms is on the order of $L / \Delta^{2}$ while the second term is on the order of $L / \Delta l_{\mathbf{n}}$, with its ratio to the first one being $\Delta / l_{\mathbf{n}} \ll 1$. Actually $l_{\mathbf{n}} \gg \Delta$ is just one of the conditions for the EL theory to be recovered as we will see below. Then, by dropping the $\partial_{k}\left(n_{i} n_{j}\right)$ term in Eq. (23) and using $\mathcal{N}_{i j}=\dot{S}\left(3 n_{i} n_{j}-\delta_{i j}\right) / 2+3 S\left(n_{i} N_{j}+n_{j} N_{i}\right) / 2$ and Eq. (21) for the definition of $h_{i j}^{\prime}$, we obtain for $Q_{i j} h_{i j}=$ $-Q_{i j} h_{i j}^{\prime}$ the equation

$$
-\frac{\partial \mathcal{F}_{L d G}^{U}}{\partial S}+\partial_{k} \frac{\partial \mathcal{F}_{L d G}^{U}}{\partial\left(\partial_{k} S\right)}=\frac{3}{2} \mu_{1} \dot{S}+\frac{3}{4} \mu_{2} n_{i} n_{j} A_{i j} .
$$

From Eq. (24), it can be deduced that the slow spatial and temporal variation of $\mathbf{n}$ effectively makes itself the only dynamic variable out of the original $\overleftrightarrow{Q}$. To see this point, we write Eq. (24) in the dimensionless form

$$
\left\{-\frac{\partial}{\partial \bar{S}}+\bar{\partial}_{k} \frac{\partial}{\partial\left(\bar{\partial}_{k} \bar{S}\right)}\right\}\left(\overline{\mathcal{F}}_{S}+\overline{\mathcal{F}}_{\mathbf{n} S}\right)=\frac{3}{2} \bar{\mu}_{1} \frac{d}{d \bar{t}} \bar{S}+\frac{3}{4} \bar{\mu}_{2} n_{i} n_{j} \bar{A}_{i j} .
$$

Here $\overline{\mathcal{F}}_{S}+\overline{\mathcal{F}}_{\mathbf{n} S}=\mathcal{F}_{L d G}^{U} /\left(B^{4} / C^{3}\right)$ is the dimensionless free energy density, $\bar{S}=S /(B / C)$ is the scaled orientational order, $\bar{\mu}_{1}=\mu_{1} / \eta$ and $\bar{\mu}_{2}=\mu_{2} / \eta$ are the dimensionless viscosity coefficients, respectively, and $\Delta$ and $\tau$ are chosen as the length and time units such that $\bar{\partial}_{k}=\Delta \partial_{k}, d / d \bar{t}=\tau(d / d t)$, and $\bar{A}_{i j}=\tau A_{i j} . \overline{\mathcal{F}}_{S}$ and $\overline{\mathcal{F}}_{\mathbf{n} S}$ are given by

$\overline{\mathcal{F}}_{S}=\left(\bar{T}+\frac{1}{4}\right) \bar{S}^{2}-\bar{S}^{3}+\bar{S}^{4}+\frac{3}{4}\left(\bar{L}_{1}+\frac{1}{6} \bar{L}_{2}\right)[\bar{\nabla} \bar{S}]^{2}+\frac{3}{8} \bar{L}_{2}[\mathbf{n} \cdot \bar{\nabla} \bar{S}]^{2}$,

and

$$
\begin{aligned}
\overline{\mathcal{F}}_{\mathbf{n} S}= & \frac{3}{2} \bar{L}_{2} \bar{S}[\bar{\nabla} \cdot \mathbf{n}][\mathbf{n} \cdot \bar{\nabla} \bar{S}]+\frac{3}{4} \bar{L}_{2} \bar{S}[\mathbf{n} \times \bar{\nabla} \times \mathbf{n}] \cdot \bar{\nabla} \bar{S} \\
& +\frac{9}{4} \bar{S}^{2}\left\{\left(\bar{L}_{1}+\frac{1}{2} \bar{L}_{2}\right)[\bar{\nabla} \cdot \mathbf{n}]^{2}+\bar{L}_{1}[\mathbf{n} \cdot \bar{\nabla} \times \mathbf{n}]^{2}\right. \\
& \left.+\left(\bar{L}_{1}+\frac{1}{2} \bar{L}_{2}\right)[\mathbf{n} \times \bar{\nabla} \times \mathbf{n}]^{2}\right\},
\end{aligned}
$$

where $\bar{T}=a C\left(T-T_{I N}\right) / B^{2}$ with the isotropic-nematic transition temperature $T_{I N}$ given by $T^{*}+B^{2} / 4 a C, \bar{L}_{1}=L_{1} / L$, and $\bar{L}_{2}=L_{2} / L . \overline{\mathcal{F}}_{S}$ is determined by the degree of orientational order $S$ and its spatial variation, while $\overline{\mathcal{F}}_{\mathbf{n} S}$ comes from the coupling between $\partial_{k} \mathbf{n}$ and $S$. Let $l_{\mathbf{n}}$ denote the characteristic distance over which the director variations take place. It follows that $\bar{\nabla} \cdot \mathbf{n}$ and $\bar{\nabla} \times \mathbf{n}$ are on the order of $\Delta / l_{\mathbf{n}}$. The slow spatial variation of $\mathbf{n}$ is defined to be $l_{\mathbf{n}} \gg \Delta$, thus $|\bar{\nabla} \cdot \mathbf{n}|,|\bar{\nabla} \times \mathbf{n}| \rightarrow 0$. As a consequence, $\overline{\mathcal{F}}_{\mathbf{n} S}$ is negligible as compared to $\overline{\mathcal{F}}_{S}$. Now let $\tau_{\mathbf{n}}$ denote the time scale associated with the dynamics of $\mathbf{n}$ and the flow. It follows that $\bar{A}_{i j}$ is on the order of $\tau / \tau_{\mathbf{n}}$. The slowness of time evolution means $\tau_{\mathbf{n}} \gg \tau$, thus $\bar{A}_{i j} \rightarrow 0$. By neglecting $\overline{\mathcal{F}}_{\mathbf{n} S}$ and $\bar{A}_{i j}$ in Eq. (25), we obtain

$$
\left\{-\frac{\partial}{\partial \bar{S}}+\bar{\partial}_{k} \frac{\partial}{\partial\left(\bar{\partial}_{k} \bar{S}\right)}\right\} \overline{\mathcal{F}}_{S}=\frac{3}{2} \bar{\mu}_{1} \frac{d}{d \bar{t}} \bar{S} .
$$

Thus the temporal variation of $\bar{S}$ is decoupled from the temporal variation of $\mathbf{n}$. In addition, we note that the equilibrium solution of $\bar{S}$ is given by the solution to the Euler-Lagrange equation 


$$
\left\{-\frac{\partial}{\partial \bar{S}}+\bar{\partial}_{k} \frac{\partial}{\partial\left(\bar{\partial}_{k} \bar{S}\right)}\right\} \overline{\mathcal{F}}_{S}=0 .
$$

Thus Eq. (28) describes the temporal relaxation of $\bar{S}$ when it is out of equilibrium. Moreover, since the response time scale $\tau$ for $S$ is much shorter than that for $\mathbf{n}$, the $\bar{S}(\mathbf{r} / \Delta, \bar{t})$ solution may be approximately given by the instantaneous optimal configuration, which minimizes the free energy $\int d(\mathbf{r} / \Delta) \overline{\mathcal{F}}_{S}[\mathbf{n}(\mathbf{r} / \Delta, \bar{t})]+\int d\left(\mathbf{S} / \Delta^{2}\right) U[\mathbf{n}(\mathbf{r} / \Delta, \bar{t})]$, noted to depend on $\mathbf{n}$ explicitly. Here $\int d \mathbf{S} U$ is the substrate-LC interaction potential. Through time-varying $\mathbf{n}$, the optimal solution $\bar{S}_{b}$ thus obtained depends on $\bar{t}$ explicitly. That means $\partial \bar{S}_{b} / \partial \bar{t}$ is on the order of $\tau / \tau_{\mathbf{n}} \rightarrow 0$. Meanwhile, $\mathbf{v}$ in the boundary layer is on the order of $\Delta \cdot \partial_{k} \mathbf{v} \sim \Delta / \tau_{\mathbf{n}}$ and $\nabla \bar{S}_{b}$ $\sim \Delta^{-1}$, thus $\tau \mathbf{v} \cdot \nabla \bar{S}_{b} \sim \tau / \tau_{\mathbf{n}}$ also tends to vanish. With $d \bar{S}_{b} / d \bar{t} \rightarrow 0$ justified, the instantaneous optimal configuration $\bar{S}_{b}$, satisfying Eq. (29) but subject to the time-varying $\mathbf{n}$ configuration, is thus obtained as the approximate solution of Eq. (28) for the boundary layer affected by the substrate-LC intercation.

Based on the above discussion, it is readily seen that the degree of orientational order $S$, varying spatially in the boundary layer but uniform in the bulk, only adiabatically follows the slow temporal variation of $\mathbf{n}$. The flow field thus induced is on the order of $\Delta / \tau_{\mathbf{n}}$, which is negligible compared with the $\mathbf{n}$-induced fluid velocity $\sim l_{\mathbf{n}} / \tau_{\mathbf{n}}$. We conclude that in the regime of $\Delta \ll l_{\mathbf{n}}$ and $\tau \ll \tau_{\mathbf{n}}$, the alignment state can be descibed by $\mathbf{n}$ alone, and $\mathbf{n}$ and $\mathbf{v}$ can be chosen as the two dynamic variables. This constitutes the basis of the EL theory, and $l_{\mathbf{n}}, \tau_{\mathbf{n}}$ may be identified with $d, \tau_{0}$ and/or $\xi_{E}, \tau_{E}$. Through a more complete discussion based on $Q_{i j}$ $=S\left(3 n_{i} n_{j}-\delta_{i j}\right) / 2+P\left(l_{i} l_{j}-m_{i} m_{j}\right) / 2$, with $\mathbf{l}, \mathbf{m}$, and $\mathbf{n}$ being the principal axes of $\overleftrightarrow{Q}$, it can be shown in a similar way that in the regime of $\Delta \ll l_{\mathbf{n}}$ and $\tau \ll \tau_{\mathbf{n}}, \mathbf{n}$ is effectively the only dynamic variable from $\overleftrightarrow{Q}$.

\section{NUMERICAL RESULTS}

We have carried out numerical calculation for a thin cell subject to a high switching voltage in order to demonstrate the LCO hydrodynamic effects. The coordinate system is defined with the $z$ axis being the substrate normal, and all quantities are functions of only $z$ and $t$. We focus on the in-plane motions with $v_{3}=0$. The boundary condition is that the velocity components $v_{1}$ and $v_{2}$ must vanish at the surfaces $z=0$ and $z=d$ ( $d$ is the cell thickness) and the surface order parameters are fixed (strong anchoring).

With $Q_{i j}=Q_{i j}(z)$ and $\mathbf{E}$ is in the $z$ direction, we have $\sigma_{31}^{d}=\sigma_{32}^{d}=0$ and $\sigma_{31}^{f}=\sigma_{32}^{f}=0$. Since the inertial terms are negligible, we obtain

$$
\begin{gathered}
\partial_{3} \sigma_{3 i}^{\prime}=0, \quad i=1,2, \\
f_{k}=-f_{k}^{\prime}, \quad k=1,2,3,4,5,
\end{gathered}
$$

where $f_{k}$ are defined by $f_{1}=h_{11}-h_{33}, f_{2}=h_{22}-h_{33}, f_{3}$ $=h_{12}+h_{21}, f_{4}=h_{23}+h_{23}$, and $f_{5}=h_{31}+h_{13}$, and $f_{k}^{\prime}$ are defined similarly. Here we select five independent order param- eters $q_{1} \equiv Q_{11}, \quad q_{2} \equiv Q_{22}, \quad q_{3} \equiv Q_{12}, \quad q_{4} \equiv Q_{23}, \quad$ and $q_{5}$ $\equiv Q_{31}$, while $f_{k}$ and $f_{k}^{\prime}$ are the molecular fields conjugate to $q_{k}$. Explicit expressions for all the quantities in Eq. (30) are given in Appendix C. The seven coupled differential equations in Eq. (30) can be integrated to yield the solution $v_{i}(z, t)$ and $q_{k}(z, t)$ provided the initial configuration $q_{k}(z, 0)$ is given. Due to the neglect of inertial terms, $v_{i}(z, 0)$ and $\dot{q}_{k}(z, 0)$ are determined by $q_{k}(z, 0)$. To determine the initial configuration, which is assumed to be in static equilibrium, we use the condition of $f_{k}=0$, i.e.,

$$
\left(-\frac{\partial}{\partial q_{k}}+\partial_{i} \frac{\partial}{\partial q_{k, i}}\right)\left(\mathcal{F}_{L d G}+\mathcal{F}_{f}\right)=0
$$

Dynamics is obtained by straightforward time integration from the initial configuration.

The cell thickness $d$ used in our computation is $100 \Delta$, on the order of 0.5 microns. $\epsilon_{a}$ is chosen to be 15 , with $\epsilon_{\|}$ $=20$ and $\epsilon_{\perp}=5$, typical for the LC's of large dielectric anisotropy. The voltage unit used here is $V_{F}=\sqrt{4 \pi K_{1} / \epsilon_{a}}$, on the order of the threshold voltage of the Fréedericksz transition. For $K_{1} \sim 10^{-6}$ dyn and $\epsilon_{a}=15, V_{F} \sim 0.3 \mathrm{~V}$, we calculate the temporally varying flow field in the cell right after a high holding voltage $V_{H}$ is switched off. The material constants and boundary conditions are given in Appendix D. Strong anchoring condition for the homogeneous alignment at the two substrates is assumed. The pretilt angle is set to be $8^{\circ}$ and the substrates are arranged to have parallel surface directors. The surface value of $S$ is fixed at $S_{0}=B(3$ $+\sqrt{33}) / 8 C$ at temperature $T=T_{I N}-4\left(T_{I N}-T^{*}\right)$. According to the EL theory, switching off $V_{H}$ results in a fast rotation of the directors close to the substrates as the large elastic distortion energy in the boundary layers is suddenly released [16]. A large flow field is thus induced by the coupled evolution of $\mathbf{n}$ and $\mathbf{v}$. This is known as the dynamic backflow effect. In particular, the EL theory gives $\tau_{E} \sim \eta \xi_{E}^{2} / K_{1}$ (here $\left.\xi_{E}=\sqrt{4 \pi K_{1} d^{2} / \epsilon_{a} V_{H}^{2}}\right)$ as the time scale of the relaxation process right after $V_{H}$ is switched off.

The calculation is performed for $V_{H}$ ranging from $10 V_{F}$ to $100 V_{F}$ (for parameters suitable to $5 \mathrm{CB}, V_{F} \simeq 0.25 \mathrm{~V}$ ). Note that $V_{F} d=V_{H} \xi_{E}$, thus $\xi_{E}$ approximately ranges from $10 \Delta$ $\left(V_{H}=10 V_{F}\right)$ to $\Delta\left(V_{H}=100 V_{F}\right)$. First we want to show that our formulation does agree with the EL theory in the regime of low voltages. This is achieved by examining the numerical results obtained for $V_{H} \sim 10 V_{F}$. Let $\mathbf{v}_{Q}$ denote the timedependent velocity fields obtained from our generalized hydrodynamic equations and $\mathbf{v}_{\mathbf{n}}$ denote that from the EL theory. In Fig. 1, it is seen that the initial $\mathbf{v}_{Q}$ and $\mathbf{v}_{\mathbf{n}}$ possess similar spatial variation but differ appreciably in magnitude. However, the initial difference vanishes quickly as $\mathbf{v}_{Q}$ approaches $\mathbf{v}_{\mathbf{n}}$ in a very short relaxation time scale $\tau$. Subsequently, $\mathbf{v}_{Q}$ merges with $\mathbf{v}_{\mathbf{n}}$ and undergoes futher relaxation with the time scale $\tau_{E}$. As the initial difference between $\mathbf{v}_{Q}$ and $\mathbf{v}_{\mathbf{n}}$ lasts only within a negligible time duration $\left(\tau \ll \tau_{E}\right)$, the two solutions are thus equivalent and the EL theory is effectively applicable. 

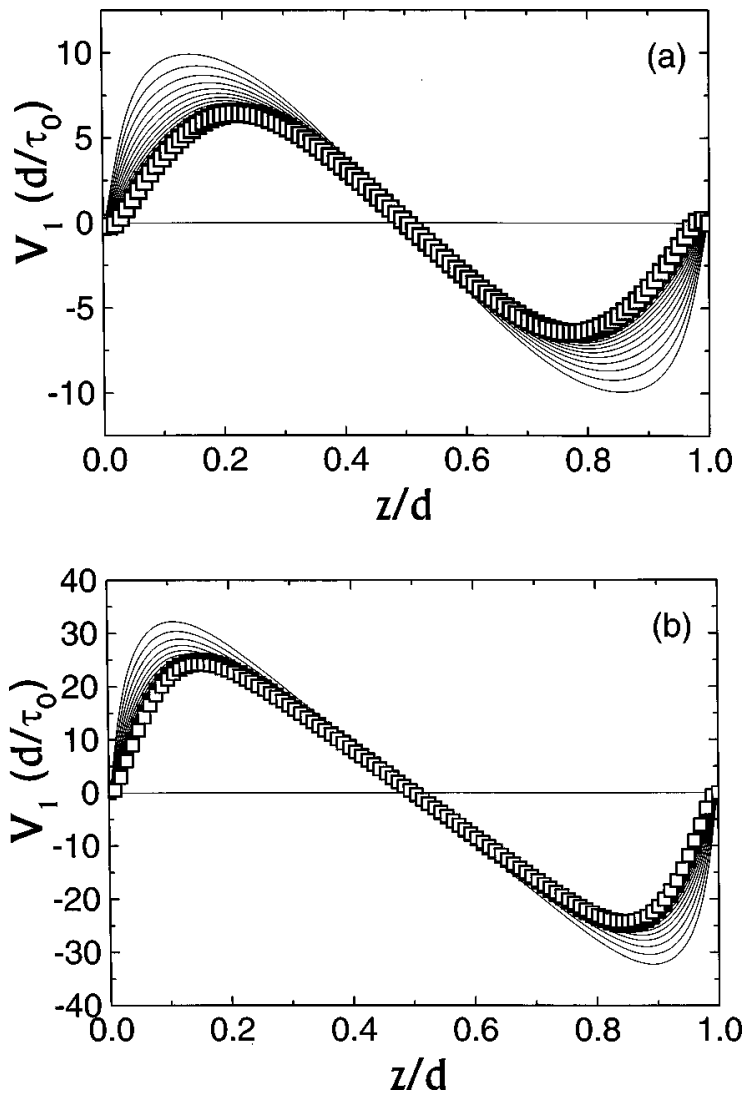

FIG. 1. (a) Spatial and temporal variations of $v_{1}(z, t)$ after the holding voltage $V_{H}=10 V_{F}$ is switched off at $t=0$. The solid lines represent the solution from the generalized formulation and the open squares represent that from the EL theory. Both solutions are obtained for the time duration from $t=0$ to $2 \times 10^{-5} \tau_{0}$, with $\tau_{0}$ $=\alpha_{4} d^{2} / K_{1}$. The time interval between each two adjacent solid lines is $2 \times 10^{-6} \tau_{0}$. It is readily seen that the temporal variation depicted by the solid lines is fast and monotonic, exhibiting a decreasing $\left|v_{1}\right|$ magnitude everywhere. For the open squares, they have similar spatial dependence but negligible temporal variation. (b) Same as (a) except for $V_{H}=20 V_{F}$.

For $V_{H}$ high enough so that $\xi_{E}$ is comparable to $\Delta$, qualitative differences emerge between our formulation and the EL theory. First, we note that within the LdG theory, the equilibrium LCO configuration (maintained by the holding voltage $V_{H}$ ) possesses strong biaxial character if $\xi_{E}$ is on the order of $\Delta$. With an initial state already beyond the director description, it is imperative to use the generalized hydrodynamic equations to solve the time evolution problem. This is further justified by considering the dynamic time scales. Since $\tau_{E}$ is actually comparable to the intrinsic time scale $\tau$ if $\xi_{E} \sim \Delta$, the dynamic relaxation involves the coupled evolution of $\overleftrightarrow{Q}$ and $\mathbf{v}$ from the very beginning. This is indeed confirmed by our numerical results. In Fig. 2, it is seen that the initial $\mathbf{v}_{Q}$ and $\mathbf{v}_{\mathbf{n}}$ still possess similar spatial variation but again are appreciably different in their magnitudes. But now the difference persists as $\mathbf{v}_{Q}$ and $\mathbf{v}_{\mathbf{n}}$ evolve, respectively, with the time scales $\tau$ and $\tau_{E}$, which are of the same order. In addition, since $\partial_{z} \mathbf{v}$ in the central part of the cell is crucial in determining the relaxational behavior of the alignment state $[16,17]$, the whole dynamic process predicted from our hydrodynamic equations differs qualitatively from that ob-
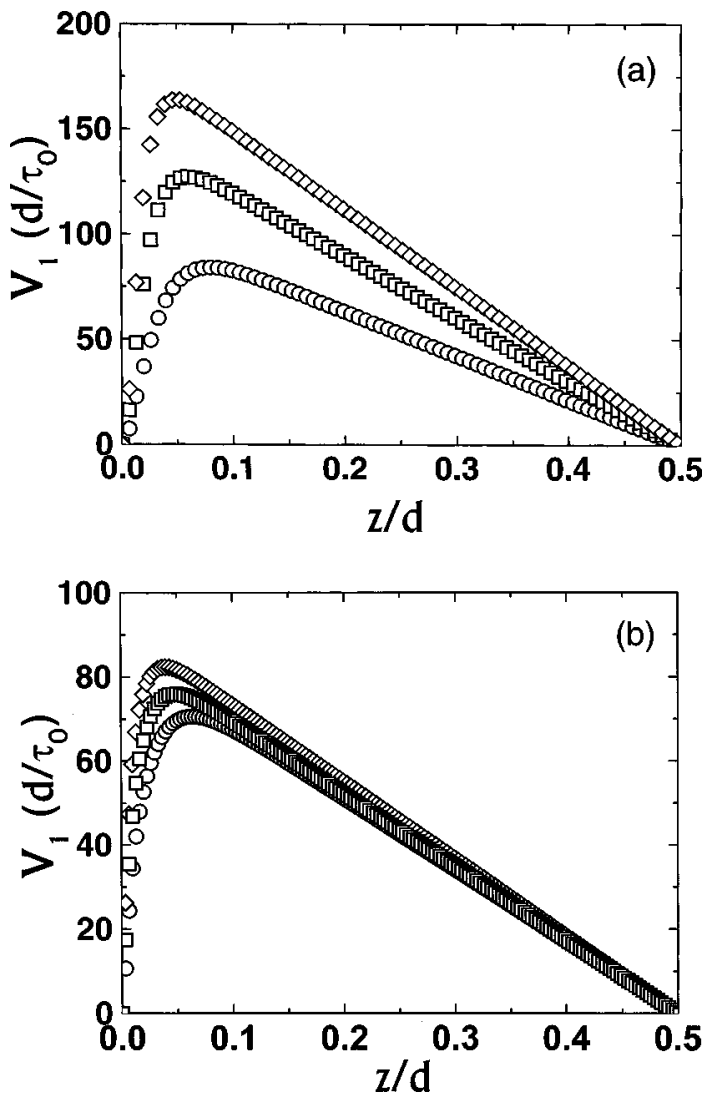

FIG. 2. (a) Initial velocity fields $v_{1}(z, t=0)$ obtained from the EL theory for $V_{H}=50 V_{F}$ (circles), $75 V_{F}$ (squares), and $100 V_{F}$ (diamonds). (b) Initial velocity fields $v_{1}(z, t=0)$ obtained from the generalized formulation for $V_{H}=50 V_{F}$ (circles), $75 V_{F}$ (squares), and $100 V_{F}$ (diamonds). Here the spatial dependence of $v_{1}$ is only depicted in the first half-space because of the symmetry relation $v_{1}(z, t)=-v_{1}(d-z, t)$.

tained within the EL theory. From Fig. 3, we see that for $V_{H} \geqslant 50 V_{F}(\simeq 13 \mathrm{~V})$, the initial velocity increase (with $\left.V_{H}\right)$ is almost flat, in sharp contrast to that predicted by the EL theory. This is due to the fact that the elastic distortion in the boundary layers is no longer characterized by the spatially rotating director. Instead, it exhibits predominant biaxial or-

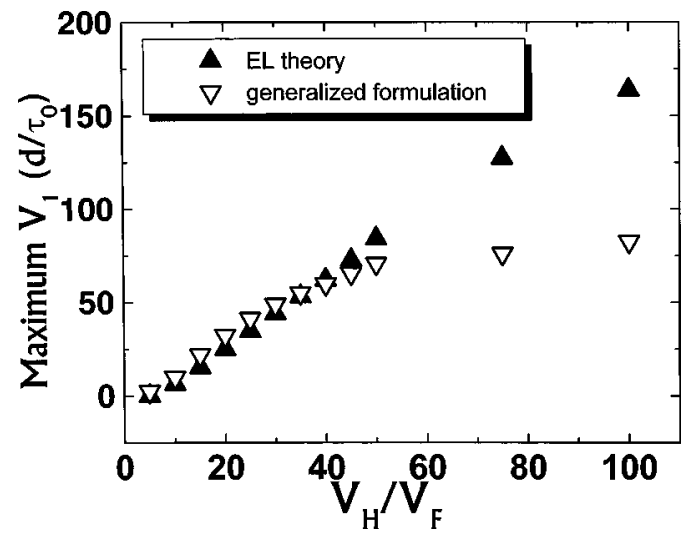

FIG. 3. Maximum initial velocity depicted as a function of the switched off $V_{H}$. Solid triangles represent the solutions from the EL theory and the open triangles represent those from the generalized formulation. 


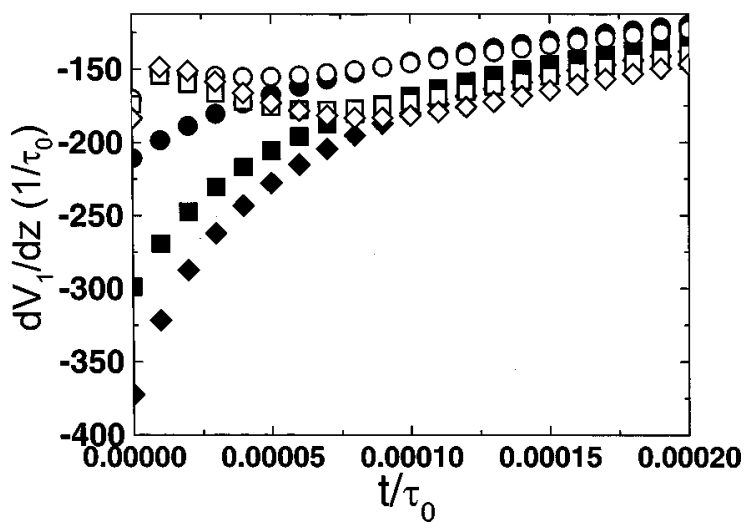

FIG. 4. Time variation of the central velocity gradient $\partial v(z$ $=d / 2, t)$ plotted as a function of time. $V_{H}=50 V_{F}$ (circles), $75 V_{F}$ (squares), and $100 V_{F}$ (diamonds) are switched off at $t=0$. The solid symbols represent the solutions from the EL theory and the open symbols represent those from the generalized formulation. It is seen that after about $\tau_{E}=10^{-4} \tau_{0}$, each solution from the generalized formulation merges with the corresponding EL solution for the same $V_{H}$.

dering with only a small rotation of the principal axes of $\overleftrightarrow{Q}$. As a result, we find the subsequent relaxation of the velocity fields to be much slower than that predicted by the EL theory, as depicted in Fig. 4.

\section{CONCLUDING REMARKS}

We have obtained the generalized hydrodynamic equations based on the tensor order parameter. Numerical results obtained for the backflow phenomena serve to make apparent the new length and time scales inherent to our formulation and to show qualitatively different dynamics in thin cells at high holding voltages. This could be important for the simulations of twisted-nematic and super-twisted-nematic cells. Further developments in taking into account more realistic surface anchoring conditions are currently under way. It is also interesting to take into account the flexoelectric effect which can be induced by either director distortion or order parameter variation [23].

\section{ACKNOWLEDGMENTS}

Tiezheng Qian would like to thank Professor Yuen-Ron Shen at Berkeley for helpful discussions. This work was supported by Hong Kong ITDC Grant No. AF/155/94 and NSF Grant No. DMR-9704384.

\section{APPENDIX A: THE ERICKSEN-LESLIE THEORY}

Based on the director description of alignment state, the Frank free energy density is given by

$\mathcal{F}_{F}=\frac{1}{2} K_{1}(\nabla \cdot \mathbf{n})^{2}+\frac{1}{2} K_{2}\left(\mathbf{n} \cdot \nabla \times \mathbf{n}+\frac{2 \pi}{P}\right)^{2}+\frac{1}{2} K_{3}(\mathbf{n} \times \nabla \times \mathbf{n})^{2}$,

where $K_{1}, K_{2}$, and $K_{3}$ are the splay, twist, and bend elastic constants, respectively, and $P$ is the helical pitch, positive for the right-handed helix. For incompressible nematics, the basic equations of the EL theory read

$$
\begin{gathered}
\partial_{i} v_{i}=0, \\
\rho \frac{d v_{i}}{d t}=\partial_{j}\left(-p \delta_{j i}+\tilde{\sigma}_{j i}^{d}+\tilde{\sigma}_{j i}^{f}+\tilde{\sigma}_{j i}^{\prime}\right), \\
I \frac{d^{2} n_{i}}{d t^{2}}=g_{i}+g_{i}^{\prime}-\tilde{\lambda} n_{i},
\end{gathered}
$$

where $\rho$ is the density constant, $p$ is the pressure, $\tilde{\boldsymbol{\sigma}}^{d}, \tilde{\boldsymbol{\sigma}}^{f}$, and $\tilde{\boldsymbol{\sigma}}^{\prime}$ are the stress tensors induced by elastic distortions, external electric and magnetic field, and viscosity effects, respectively, $I$ is the inertial momentum density that is usually negligible, $\mathbf{g}$ and $\mathbf{g}^{\prime}$ are the elastic and viscous molecular fields, and $\tilde{\lambda}$ is the Lagrange multiplier imposed by $\mathbf{n}^{2}=1$. $\tilde{\boldsymbol{\sigma}}^{d}$ and $\mathbf{g}$ are determined by the Frank free energy through

$$
\begin{gathered}
\tilde{\sigma}_{j i}^{d}=-\frac{\partial \mathcal{F}_{F}}{\partial\left(\partial_{j} n_{k}\right)} \partial_{i} n_{k}, \\
g_{i}=-\frac{\partial \mathcal{F}_{F}}{\partial n_{i}}+\partial_{k} \frac{\partial \mathcal{F}_{F}}{\partial\left(\partial_{k} n_{i}\right)} .
\end{gathered}
$$

The entropy source of the isothermal, dissipative processes is given by

$$
T \dot{\Sigma}=\int d \mathbf{r}\left\{\tilde{\sigma}_{j i}^{\prime} \partial_{j} v_{i}-\mathbf{g}^{\prime} \cdot \dot{\mathbf{n}}\right\}
$$

The conservation of angular momentum relates $\mathbf{g}^{\prime}$ to the antisymmetric part of $\tilde{\boldsymbol{\sigma}}^{\prime}$, and this fact leads to the expression

$$
T \dot{\Sigma}=\int d \mathbf{r}\left\{\tilde{\boldsymbol{\sigma}}_{j i}^{\prime s} A_{j i}-\mathbf{g}^{\prime} \cdot \mathbf{N}\right\},
$$

where $\tilde{\sigma}_{j i}^{\prime s}$ is the symmetric part of $\tilde{\sigma}_{j i}^{\prime}, A_{j i}=\frac{1}{2}\left(\partial_{j} v_{i}+\partial_{i} v_{j}\right)$ is the symmetric part of the velocity gradient tensor, and $\mathbf{N}$ $=\dot{\mathbf{n}}-\boldsymbol{\omega} \times \mathbf{n}$ is time-changing rate of the director with respect to the background fluid with angular velocity $\boldsymbol{\omega}=\frac{1}{2} \nabla \times \mathbf{v}$. The independent fluxes contributing to the entropy production are thus identified. For $A_{j i}$ and $N_{i}$, which are weak on the molecular scale, the viscous stress and molecular field are linear functions of the fluxes:

$$
\begin{gathered}
\tilde{\sigma}_{j i}^{\prime}=\alpha_{1} n_{j} n_{i} n_{\mu} n_{\nu} A_{\mu \nu}+\alpha_{4} A_{j i}+\alpha_{5} n_{j} n_{\mu} A_{\mu i}+\alpha_{6} n_{i} n_{\mu} A_{\mu j} \\
+\alpha_{2} n_{j} N_{i}+\alpha_{3} n_{i} N_{j}, \\
-g_{i}^{\prime}=\gamma_{2} n_{j} A_{j i}+\gamma_{1} N_{i},
\end{gathered}
$$

where the six $\alpha_{i}$ are the Leslie coefficients, with $\gamma_{1}=\alpha_{3}$ $-\alpha_{2}$ and $\gamma_{2}=\alpha_{3}+\alpha_{2}=\alpha_{6}-\alpha_{5}$ [22].

\section{APPENDIX B: COMPARISON OF EL THEORY WITH THE GENERAL FORMULATION}

We equate the LdG free energy with the Frank free energy in the limit of Eq. (9). From the definitions (5) and 
(A6), the vector molecular field $\mathbf{g}$ is related to the tensor field h by

$$
g_{j}=\frac{3 S_{0}}{2}\left(n_{i} h_{i j}+n_{i} h_{j i}\right) .
$$

Here the field-induced terms in $\mathbf{g}$ and $\mathbf{h}$ are included because $-(1 / 12 \pi) \epsilon_{a}^{m} Q_{i j} Q_{i} E_{j}=-(1 / 8 \pi) \epsilon_{a}(\mathbf{n} \cdot \mathbf{E})^{2}+$ constant, with $\epsilon_{a}=S_{0} \epsilon_{a}^{m}$. The equilibrium condition $h_{i j}=\lambda \delta_{i j}+\epsilon_{i j k} \lambda_{k}$ is thus equivalent to $g_{i}=3 S_{0} \lambda n_{i}$ as obtained by minimizing the Frank free energy with $3 S_{0} \lambda$ being the Lagrange multiplier (due to $\mathbf{n}^{2}=1$ ). The distortion stress $\sigma_{j i}^{d}=-\Pi_{j \alpha \beta} Q_{\alpha \beta, i}$ is equal to $\tilde{\sigma}_{j i}^{d}$ with $\frac{3}{2} S_{0}\left(n_{j} \Pi_{k i j}+n_{j} \Pi_{k j i}\right)=\partial \mathcal{F}_{F} / \partial n_{i, k}$. The field-induced stresses $\boldsymbol{\sigma}^{f}$ and $\widetilde{\boldsymbol{\sigma}}^{f}$ are the same in Eq. (6) with $\stackrel{\leftrightarrow}{\boldsymbol{\epsilon}}$ expressed in terms of $\overleftrightarrow{Q}$ and $\mathbf{n}$, respectively. As for the viscous forces, $\mathbf{g}^{\prime}$ is related to $\mathbf{h}^{\prime}$ by

$$
g_{j}^{\prime}=\frac{3 S_{0}}{2}\left(n_{i} h_{i j}^{\prime}+n_{i} h_{j i}^{\prime}\right)
$$

which is of the same form as Eq. (B1). Equation (B2) and $\mathcal{N}_{i j}=3 S_{0}\left(n_{i} N_{j}+N_{i} n_{j}\right) / 2$ yields the same orientational entropy production equation $h_{i j}^{\prime} \mathcal{N}_{i j}=g_{k}^{\prime} N_{k}$. From Eqs. (B1), (B2) and $2 n_{j} \ddot{Q}_{i j}=3 S_{0} \ddot{n}_{i}+3 S_{0} n_{j} \ddot{n}_{j} n_{i}$, we see the vectorial Oseen equation (A4) can be constructed from the tensorial Eq. (3) with $I=\frac{9}{2} J S_{0}^{2}$ and $\tilde{\lambda}=3 S_{0} \lambda+\frac{9}{2} J S_{0}^{2} n_{j} \ddot{n}_{j} . I=\frac{9}{2} J S_{0}^{2}$ is also obtained from the energy relation $\frac{1}{2} I \ddot{\mathbf{n}}^{2}=\frac{1}{2} J \dot{Q}_{i j}^{2}$ or the angular momentum relation $I(\mathbf{n} \times \ddot{\mathbf{n}})_{i}=\epsilon_{i j k} J\left(Q_{j \mu} \dot{Q}_{k \mu}\right.$ $\left.+Q_{\mu j} \dot{Q}_{\mu k}\right) . \boldsymbol{\sigma}^{\prime}$ and $\tilde{\boldsymbol{\sigma}}^{\prime}$ are required to be identical in the limit of Eq. (9) since they both serve in the same dynamic equation $\rho \dot{v}_{i}=\partial_{j} \sigma_{j i}$. This fact, combined with Eq. (B2), can be used to establish the relation between the Leslie coefficients and the viscosity coefficients defined in $\boldsymbol{\sigma}^{\prime}$ and $\mathbf{h}^{\prime}$. From Eqs. (8) and (A10), we get $\gamma_{1}=\frac{9}{2} S_{0}^{2} \mu_{1}$ and $\gamma_{2}$ $=\frac{3}{2} S_{0} \mu_{2}$. Equating $\sigma_{j i}^{\prime}$ with $\tilde{\sigma}_{j i}^{\prime}$ yields

$$
\begin{gathered}
\alpha_{1}=9 S_{0}^{2} \beta_{1} / 4, \\
\alpha_{2}=3 S_{0} \mu_{2} / 4-9 S_{0}^{2} \mu_{1} / 4, \\
\alpha_{3}=3 S_{0} \mu_{2} / 4+9 S_{0}^{2} \mu_{1} / 4, \\
\alpha_{4}=\beta_{4}-S_{0}\left(\beta_{5}+\beta_{6}\right) / 2, \\
\alpha_{5}=3 S_{0} \beta_{5} / 2=3 S_{0}\left(\beta_{5}+\beta_{6}-\mu_{2}\right) / 4, \\
\alpha_{6}=3 S_{0} \beta_{6} / 2=3 S_{0}\left(\beta_{5}+\beta_{6}+\mu_{2}\right) / 4,
\end{gathered}
$$

which give $\gamma_{1}=\alpha_{3}-\alpha_{2}$ and $\gamma_{2}=\alpha_{6}-\alpha_{5}$ with the Parodi relation $\alpha_{3}+\alpha_{2}=\alpha_{6}-\alpha_{5}$. The six Leslie coefficients linked by the Parodi relation can be used to completely determine the value of six viscosity coefficients $\beta_{1}, \beta_{4}, \beta_{5}, \beta_{6}, \mu_{1}$, and $\mu_{2}$ linked by $\beta_{6}-\beta_{5}=\mu_{2}$, provided $S_{0}$ is known. Note that other terms in higher orders of $Q_{i j}$ can be added to the expansions of $\sigma_{i j}^{\prime s}$ and $h_{i j}^{\prime}$ in Eqs. (20) and (21). However, additional viscosity coefficients would have to be introduced $[14,15]$, and they can not be determined by the Leslie coefficients. In summary, our formulation can be reduced to the EL theory with the relations (B1), (B2), $\boldsymbol{\sigma}^{d}=\tilde{\boldsymbol{\sigma}}^{d}, \boldsymbol{\sigma}^{f}=\tilde{\boldsymbol{\sigma}}^{f}$, and $\boldsymbol{\sigma}^{\prime}=\tilde{\boldsymbol{\sigma}}^{\prime}$ established in the limit of homogeneous, uniaxial ordering with uniform $S=S_{0}$.

\section{APPENDIX C: EXPLICIT EXPRESSIONS}

For planar fluid motion $\left(v_{3}=0\right)$, we only need to use $\sigma_{31}^{\prime}$ and $\sigma_{32}^{\prime}$. From Eq. (7), we have

$$
\begin{aligned}
\sigma_{31}^{\prime}= & \beta_{1} Q_{31} Q_{\mu \nu} A_{\mu \nu}+\beta_{4} A_{31}+\beta_{5} Q_{3 \mu} A_{\mu 1}+\beta_{6} Q_{1 \mu} A_{\mu 3} \\
& +\frac{1}{2} \mu_{2} N_{31}-\mu_{1} Q_{3 \mu} N_{\mu 1}+\mu_{1} Q_{1 \mu} N_{\mu 3}, \\
\sigma_{32}^{\prime}= & \beta_{1} Q_{32} Q_{\mu \nu} A_{\mu \nu}+\beta_{4} A_{32}+\beta_{5} Q_{3 \mu} A_{\mu 2}+\beta_{6} Q_{2 \mu} A_{\mu 3} \\
& +\frac{1}{2} \mu_{2} N_{32}-\mu_{1} Q_{3 \mu} N_{\mu 2}+\mu_{1} Q_{2 \mu} N_{\mu 3} .
\end{aligned}
$$

Introducing the coefficients $U_{k i}$ and $V_{k i}$ with $\sigma_{3 k}=U_{k i} \dot{q}_{i}$ $+V_{k j} v_{j}^{\prime}(k=1,2, i=1,2,3,4,5$, and $j=1,2)$, we have

$$
\begin{gathered}
U_{11}=-2 \mu_{1} q_{5}, \quad U_{12}=-\mu_{1} q_{5}, \quad U_{13}=-\mu_{1} q_{4}, \quad U_{14}=\mu_{1} q_{3}, \quad U_{15}=\mu_{1}\left(2 q_{1}+q_{2}\right)+\frac{1}{2} \mu_{2}, \\
V_{11}=\beta_{1} q_{5}^{2}+\frac{1}{2}\left(\beta_{4}-\beta_{5}\left(q_{1}+q_{2}\right)+\beta_{6} q_{1}\right)+\frac{1}{4} \mu_{2}\left(2 q_{1}+q_{2}\right)+\frac{1}{2} \mu_{1}\left(q_{3}^{2}+q_{4}^{2}+4 q_{5}^{2}+\left(2 q_{1}+q_{2}\right)^{2}\right), \\
V_{12}=\beta_{1} q_{4} q_{5}+\frac{1}{2} \beta_{6} q_{3}+\frac{1}{4} \mu_{2} q_{3}+\frac{3}{2} \mu_{1}\left(q_{4} q_{5}+\left(q_{1}+q_{2}\right) q_{3}\right), \\
U_{21}=-\mu_{1} q_{4}, \quad U_{22}=-2 \mu_{1} q_{4}, \quad U_{23}=-\mu_{1} q_{5}, \quad U_{24}=\mu_{1}\left(q_{1}+2 q_{2}\right)+\frac{1}{2} \mu_{2}, \quad U_{25}=\mu_{1} q_{3}, \\
V_{21}=\beta_{1} q_{4} q_{5}+\frac{1}{2} \beta_{6} q_{3}+\frac{1}{4} \mu_{2} q_{3}+\frac{3}{2} \mu_{1}\left(q_{4} q_{5}+\left(q_{1}+q_{2}\right) q_{3}\right), \\
V_{22}=\beta_{1} q_{4}^{2}+\frac{1}{2}\left(\beta_{4}-\beta_{5}\left(q_{1}+q_{2}\right)+\beta_{6} q_{2}\right)+\frac{1}{4} \mu_{2}\left(q_{1}+2 q_{2}\right)+\frac{1}{2} \mu_{1}\left(q_{3}^{2}+4 q_{4}^{2}+q_{5}^{2}+\left(q_{1}+2 q_{2}\right)^{2}\right) .
\end{gathered}
$$

Here $\dot{q}_{i}=\partial_{t} q_{i}$ and $v_{j}^{\prime} \equiv \partial_{z} v_{j}$. The expressions for $f_{k}^{\prime}$ are relatively simple. From Eq. (8), we have 


$$
\begin{gathered}
-f_{1}^{\prime}=-h_{11}^{\prime}+h_{33}^{\prime}=2 \mu_{1}\left(\dot{q}_{1}-v_{1}^{\prime} q_{5}\right)+\mu_{1}\left(\dot{q}_{2}-v_{2}^{\prime} q_{4}\right), \\
-f_{2}^{\prime}=-h_{22}^{\prime}+h_{33}^{\prime}=2 \mu_{1}\left(\dot{q}_{2}-v_{2}^{\prime} q_{4}\right)+\mu_{1}\left(\dot{q}_{1}-v_{1}^{\prime} q_{5}\right), \\
-f_{3}^{\prime}=-h_{12}^{\prime}-h_{21}^{\prime}=\mu_{1}\left(2 \dot{q}_{3}-v_{1}^{\prime} q_{4}-v_{2}^{\prime} q_{5}\right), \\
-f_{4}^{\prime}=-h_{23}^{\prime}-h_{32}^{\prime}=\frac{1}{2} \mu_{2} v_{2}^{\prime}+\mu_{1}\left(2 \dot{q}_{4}+v_{1}^{\prime} q_{3}+v_{2}^{\prime}\left(q_{1}+2 q_{2}\right)\right), \\
-f_{5}^{\prime}=-h_{31}^{\prime}-h_{13}^{\prime}=\frac{1}{2} \mu_{2} v_{1}^{\prime}+\mu_{1}\left(2 \dot{q}_{5}+v_{2}^{\prime} q_{3}+v_{1}^{\prime}\left(2 q_{1}+q_{2}\right)\right) .
\end{gathered}
$$

Introducing another set of coefficients $u_{k i}$ and $v_{k j}$ with $f_{k}^{\prime}=u_{k i} \dot{q}_{i}+v_{k j} v_{j}^{\prime}(k, i=1,2,3,4,5$ and $j=1,2)$, we have

$$
\begin{gathered}
u_{11}=2 \mu_{1}, \quad u_{12}=\mu_{1}, \quad u_{13}=0, \quad u_{14}=0, \quad u_{15}=0, \quad v_{11}=-2 \mu_{1} q_{5}, \quad v_{12}=-\mu_{1} q_{4}, \\
u_{21}=\mu_{1}, \quad u_{22}=2 \mu_{1}, \quad u_{23}=0, \quad u_{24}=0, \quad u_{25}=0, \quad v_{21}=-\mu_{1} q_{5}, \quad v_{22}=-2 \mu_{1} q_{4}, \\
u_{31}=0, \quad u_{32}=0, \quad u_{33}=2 \mu_{1}, \quad u_{34}=0, \quad u_{35}=0, \quad v_{31}=-\mu_{1} q_{4}, \quad v_{32}=-\mu_{1} q_{5}, \\
u_{41}=0, \quad u_{42}=0, \quad u_{43}=0, \quad u_{44}=2 \mu_{1}, \quad u_{45}=0, \quad v_{41}=\mu_{1} q_{3}, \quad v_{42}=\frac{1}{2} \mu_{2}+\mu_{1}\left(q_{1}+2 q_{2}\right), \\
u_{51}=0, \quad u_{52}=0, \quad u_{53}=0, \quad u_{54}=0, \quad u_{55}=2 \mu_{1}, \quad v_{51}=\frac{1}{2} \mu_{2}+\mu_{1}\left(2 q_{1}+q_{2}\right), \quad v_{52}=\mu_{1} q_{3} .
\end{gathered}
$$

Using the free energy expression and the definitions of $h_{i j}$ and $f_{k}$, we obtain

$$
\begin{gathered}
f_{1}=-\alpha\left(2 q_{1}+q_{2}\right)+3 \beta\left(q_{1}^{2}+q_{3}^{2}-q_{5}^{2}-\left(q_{1}+q_{2}\right)^{2}\right)-4 \gamma\left(2 q_{1}+q_{2}\right) R+L_{1}\left(2 q_{1}^{\prime \prime}+q_{2}^{\prime \prime}\right)+L_{2}\left(q_{1}^{\prime \prime}+q_{2}^{\prime \prime}\right)+2 \pi L_{1}\left(4 q_{3}^{\prime}\right) / P \\
-\frac{\epsilon_{a}^{m}}{12 \pi} \frac{V^{2}}{\epsilon_{33}^{2}\left(\int_{0}^{d} d z / \epsilon_{33}\right)^{2}}, \\
f_{2}=-\alpha\left(q_{1}+2 q_{2}\right)+3 \beta\left(q_{2}^{2}+q_{3}^{2}-q_{5}^{2}-\left(q_{1}+q_{2}\right)^{2}\right)-4 \gamma\left(q_{1}+2 q_{2}\right) R+L_{1}\left(q_{1}^{\prime \prime}+2 q_{2}^{\prime \prime}\right)+L_{2}\left(q_{1}^{\prime \prime}+q_{2}^{\prime \prime}\right) \\
+2 \pi L_{1}\left(-4 q_{3}^{\prime}\right) / P-\frac{\epsilon_{a}^{m}}{12 \pi} \frac{V^{2}}{\epsilon_{33}^{2}\left(\int_{0}^{d} d z / \epsilon_{33}\right)^{2}}, \\
f_{3}=-2 \alpha q_{3}+6 \beta\left(q_{1} q_{3}+q_{2} q_{3}+q_{4} q_{5}\right)-8 \gamma q_{3} R+2 L_{1} q_{3}^{\prime \prime}+2 \pi L_{1}\left(-4 q_{1}^{\prime}+4 q_{2}^{\prime}\right) / P, \\
f_{4}=-2 \alpha q_{4}+6 \beta\left(q_{2} q_{4}+q_{3} q_{5}-q_{4}\left(q_{1}+q_{2}\right)\right)-8 \gamma q_{4} R+2 L_{1} q_{4}^{\prime \prime}+L_{2} q_{4}^{\prime \prime}+2 \pi L_{1}\left(-4 q_{5}^{\prime}\right) / P, \\
f_{5}=-2 \alpha q_{5}+6 \beta\left(q_{1} q_{5}+q_{3} q_{4}-q_{5}\left(q_{1}+q_{2}\right)\right)-8 \gamma q_{5} R+2 L_{1} q_{5}^{\prime \prime}+L_{2} q_{5}^{\prime \prime}+2 \pi L_{1}\left(4 q_{4}^{\prime}\right) / P
\end{gathered}
$$

with $q_{k}^{\prime \prime} \equiv \partial_{z}^{2} q_{k}$ and $R=Q_{i j} Q_{i j}=q_{1}^{2}+q_{2}^{2}+\left(q_{1}+q_{2}\right)^{2}+2 q_{3}^{2}$ $+2 q_{4}^{2}+2 q_{5}^{2}$.

\section{APPENDIX D: MATERIAL PARAMETERS}

Here we give the numerical values of the various parameters used in our calculation. The material constants in the free energy expansion are $a=0.065 \mathrm{~J} / \mathrm{cm}^{3} \mathrm{~K}, \quad B$ $=0.53 \mathrm{~J} / \mathrm{cm}^{3}, C=0.98 \mathrm{~J} / \mathrm{cm}^{3}, L=4.5 \times 10^{-14} \mathrm{~J} / \mathrm{cm}$, and $L_{2} / L 1=1$, suitable for $5 \mathrm{CB}$ [24]. The temperature is $T$ $=T_{I N}-4\left(T_{I N}-T^{*}\right)$. The Frank elastic constants are given by $K_{1}=\frac{9}{2} S_{0}^{2}\left(L_{1}+\frac{1}{2} L_{2}\right), \quad K_{2}=\frac{9}{2} S_{0}^{2} L_{1}, \quad$ and $\quad K_{3}=\frac{9}{2} S_{0}^{2}\left(L_{1}\right.$ $\left.+\frac{1}{2} L_{2}\right)$, with $S_{0}=B(3+\sqrt{33}) / 8 C$ at temperature $T$. Since very few measured viscosity coefficients are available, here the Leslie viscosity coefficients are taken from Ref. [16], suitable for $p$-methoxybenzylidene- $p$-( $n$-butyl)aniline (MBBA). They are listed in Table I. Using Eq. (B3), the viscosity coefficients in Eq. (29) can be obtained. Other parameters are $\epsilon_{\|}=20, \epsilon_{\perp}=5, d=100 \Delta$, and $P=2 d$. The voltage unit $V_{F}=\sqrt{4 \pi K_{1} / \epsilon_{a}}$ is $0.253 \mathrm{~V}$. The pretilt angle of substrate alignment is $\theta_{s}=8^{\circ}$ and the boundary condition for $\overleftrightarrow{Q}$ is $\overleftrightarrow{Q}(0)=\overleftrightarrow{Q}(d)=S_{0}\left(3 \mathbf{n}_{s} \mathbf{n}_{s}-\mathbf{I}\right) / 2$ with $\mathbf{n}_{s}$ $=\left(\cos \theta_{s}, 0, \sin \theta_{s}\right)$.

TABLE I. The viscosity coefficients.

\begin{tabular}{lccc}
\hline \hline$\alpha_{1} / \alpha_{4}$ & 0.07808 & $\alpha_{2} / \alpha_{4}$ & -0.9315 \\
$\alpha_{3} / \alpha_{4}$ & -0.01435 & $\alpha_{4}$ & $0.4598 \mathrm{cgs}$ \\
$\alpha_{5} / \alpha_{4}$ & 0.5565 & $\alpha_{6} / \alpha_{4}$ & $\left(\alpha_{2}+\alpha_{3}+\alpha_{5}\right) / \alpha_{4}$ \\
\hline \hline
\end{tabular}


[1] C. W. Oseen, Trans. Faraday Soc. 29, 883 (1933).

[2] H. Zocher, Trans. Faraday Soc. 29, 945 (1933).

[3] F. C. Frank, Discuss. Faraday Soc. 25, 19 (1958).

[4] J. L. Ericksen, Arch. Ration. Mech. Anal. 4, 231 (1960); J. L. Ericksen, Phys. Fluids 9, 1205 (1966).

[5] F. M. Leslie, Q. J. Mech. Appl. Math. 19, 357 (1966); F. M. Leslie, Arch. Ration. Mech. Anal. 28, 265 (1969).

[6] P. G. de Gennes, Phys. Lett. A 30, 454 (1969); P. G. de Gennes, Mol. Cryst. Liq. Cryst. 12, 193 (1971).

[7] P. Sheng and E. B. Priestley, in The Landau-de Gennes Theory of Liquid Crystal Phase Transitions, Introduction to Liquid Crystals, edited by E. B. Priestley, P. J. Wojtowicz, and P. Sheng (Plenum Press, New York, 1975), p. 153.

[8] P. Sheng, Phys. Rev. A 26, 1610 (1982); T. J. Sluckin and A. Poniewierski, Phys. Rev. Lett. 55, 2907 (1985); W. Chen, L. J. Martinez-Miranda, H. Hsiung, and Y. R. Shen, ibid. 62, 1860 (1989).

[9] X. Zhuang, L. Marrucci, and Y. R. Shen, Phys. Rev. Lett. 73, 1513 (1994).

[10] T. Z. Qian and P. Sheng, Phys. Rev. Lett. 77, 4564 (1996); T. Z. Qian and P. Sheng, Phys. Rev. E 55, 7111 (1997).

[11] N. Schopohl and T. J. Sluckin, Phys. Rev. Lett. 59, 2582 (1987).

[12] P. Palffy-Muhoray, E. C. Gartland, and J. R. Kelly, Liq. Cryst. 16, 713 (1994).
[13] A. Kilian and S. Hess, Z. Naturforsch., A: Phys. Sci. 44, 693 (1989); A. Sonnet, A. Kilian, and S. Hess, Phys. Rev. E 52, 718 (1995).

[14] H. Imura and K. Okano, Jpn. J. Appl. Phys. 11, 1440 (1979).

[15] A. C. Diogo and A. F. Martins, J. Phys. (Paris) 43, 779 (1982).

[16] C. Z. van Doorn, J. Appl. Phys. 46, 3738 (1975); D. W. Berreman, ibid. 46, 3746 (1975).

[17] I. Dozov, M. Nobili, and G. Durand, Appl. Phys. Lett. 70, 1179 (1997); T. Z. Qian, Z. L. Xie, H. S. Kwok, and P. Sheng, ibid. 71, 596 (1997).

[18] L. D. Landau and E. M. Lifshitz, Electrodynamics of Continuous Media, 2nd ed. (Pergamon Press, New York, 1984).

[19] S. de Groot and P. Mazur, Non-equilibrium Thermodynamics (North-Holland, Amsterdam, 1962).

[20] In a field theory with the free energy density of the form $k(\nabla \phi)^{2}+m \phi^{2}$, the elastic correlation length is $\sqrt{k / m}$. The Frank energy only considers the gradient terms and the elastic correlation length is thus infinite.

[21] T. C. Lubensky, Phys. Rev. A 2, 2497 (1970).

[22] O. Parodi, J. Phys. (Paris) 31, 581 (1970).

[23] R. B. Meyer, Phys. Rev. Lett. 22, 918 (1969); G. Barbero, I. Dozov, J. F. Palierne, and G. Durand, ibid. 56, 2056 (1986).

[24] P. Sheng, B. Z. Li, M. Y. Zhou, T. Moses, and Y. R. Shen, Phys. Rev. A 46, 946 (1992). 